\title{
POPSIM: A Platform Targeting the Modeling and Simulation of Human Populations in Urban Environments
}

\author{
Linus J. Luotsinen \\ Swedish Defence Research Agency (FOI) \\ SE-164 90, Stockholm, Sweden \\ linus.luotsinen@foi.se
}

\begin{abstract}
In this paper we describe a simulation platform, POPSIM, targeting the modeling and simulation of human populations in urban environments. POPSIM employs an agentbased modeling approach where individuals are intuitively represented by autonomous agents in the simulated population. Our efforts thus far have resulted in a prototype platform that embeds the following core functionality: a population synthesizer responsible for generating agent specific characteristics using demographic, geographic and relationship statistics; an extensible routine behavior model that use activity statistics to generate agent plans representing daily activities such as working, eating, leisure, sleeping; a growing set of critical city infrastructure models representing buildings, road-networks and cell-phone networks; and finally, a growing set of modular and reusable agent services such as activity planning and execution, field-of-view estimation, path-planning, opinion dynamics, etc.
\end{abstract}

\section{Categories and Subject Descriptors}

I.6.5 [Simulation and Modeling]: Model Development

\section{General Terms}

Algorithms, Design

\section{Keywords}

Population simulation, urban simulation, agent-based modeling

\section{INTRODUCTION}

Human societies and cities are complex socio-technical systems that are overwhelmingly difficult for decision makers such as politicians or military leaders to embrace. Complexity arise not only from human behavior but also from the services provided by highly interconnected and interdependent infrastructure systems such as buildings, roadnetworks, power-grids, water and sewage, wireless services

Permission to make digital or hard copies of all or part of this work for personal or classroom use is granted without fee provided that copies are not made or distributed for profit or commercial advantage and that copies bear this notice and the full citation on the first page. To copy otherwise, to republish, to post on servers or to redistribute to lists, requires prior specific permission and/or a fee.

Simutools 2014, March 17-19, Lisbon, Portugal

Copyright (c) 2014 ICST 978-1-63190-007-5

DOI 10.4108/icst.simutools.2014.254628 and so on. Due to this complexity even the most experienced leaders are likely to struggle to make decisions in which unforeseen and unwanted effects are recognized and avoided in a timely and cost-efficient manner.

In this work we have developed a modular and extensible simulation platform, POPSIM, that incorporates AI techniques and algorithms to model and simulate the behavior of human populations in urban environments. POPSIM employs an agent-based modeling (ABM) approach where individuals are intuitively represented by autonomous agents in the simulated population. The long-term goal of our work is to provide a tool that can be used in simulation based training and decision support to better understand the effects of actions, policies, etc. even prior to applying them in the real-world.

Our efforts thus far have resulted in a prototype platform that embeds:

- a population synthesizer that is used to generate agent specific characteristics representing the initial state of the population using demographic, geographic and relationship statistics.

- an extensible routine behavior model that use activity statistics to generate agent plans representing daily activities such as working, eating, leisure and sleeping.

- a growing set of critical infrastructure models such as buildings, roads and mobile communication networks that provide the basic services to support the execution of agent activities within a city.

- a growing set of modular and reusable agent services such as activity planning and execution, field-of-view estimation and path-planning.

- a wide range of real-time visualization possibilities that capture the dynamics of the simulated population using maps, line- and bar-plots.

In the following sections we describe related works; the platform and its core components; practical applications; and finally, conclusions and future work.

\section{RELATED WORKS}

The dynamics and behavior of human populations can be modeled and simulated using, macroscopic and equation based, methods such as System Dynamics (SD) [10] or using microscopic entity based methods such as Agent-Based Modeling (ABM) or Cellular Automata (CA) [6]. Examples 
of macroscopic models are given in [21] and in [4]. The remainder of this section will focus on related works where the microscopic method has been used to simulate human behavior in both scientific as well as commercial applications.

Researchers within the transportation domain have for many years used agent-based simulations to analyze and plan road-network improvements or modifications within cities. The Transportation Analysis Simulation System (TRANSIMS) is a collection of research tools targeting the transportation planning problem of large cities [19]. The system has been applied to analyze traffic in several real-world cities (e.g. Dallas and Portland) and it is capable of simulating populations reaching millions of individuals using parallel and high-performance computing. Other similar tools developed within the transportation domain are MATSim [1] and VISSIM [5].

Disaster management and in particular evacuation modeling is another domain where microsimulation has been used in the past. EXODUS is one example of a framework targeting the simulation of evacuation caused by fires in buildings, maritime vehicles, airborne vehicles and rolling stock [12]. The EXODUS tool consists of modules controlling the physical movement, behavior and properties (age, gender, agility, etc.) of each individual. The tool also includes modules to calculate the dispersion of heat, smoke and toxic products and finally a module to calculate the health-effects when exposed to toxic products.

Simulation based training within the military is another domain where population or civilian models have been frequently used in the past. Commercial serious gaming products such as VBS2 and DI-Guy are capable of modeling civilian behaviors as well as their three-dimensional physical capabilities and appearances.

POPSIM builds on previous works and provides a highly extensible platform in which core functions and models can be reused to more efficiently, with respect to cost and time, generate human population models for various application domains including the ones mentioned above. This specialization separates POPSIM from other, general purpose, ABM-platforms such as MASON [17], GAMA [11], RePast, etc. Other main contributions of POPSIM are its ability to model social bonds (family, friends, etc.) to form synthetic social networks and its ability to simulate the dynamics of how opinions such as political views, attitude towards the regime or military forces, etc. may evolve over time within a population.

\section{THE POPSIM PLATFORM}

POPSIM was implemented using the Java programming language and includes, besides the core libraries, a Graphical User Interface (GUI) that builds on the Eclipse Rich Client Platform (RCP) to visualize and monitor the dynamics of simulation runs. Java was primarily employed to achieve platform independence but also to reduce development costs through reuse of the many open-source libraries that are available within the Java community. The main drawback of Java is that it is an interpretive language which ultimately results in a computational performance loss when compared to languages where code is natively compiled. In POPSIM we have addressed this issue by allowing critical components to execute natively using the Java Native Interface (JNI).

Let us now describe the main components of POPSIM. In Figure 1 the workflow of the components is presented. A

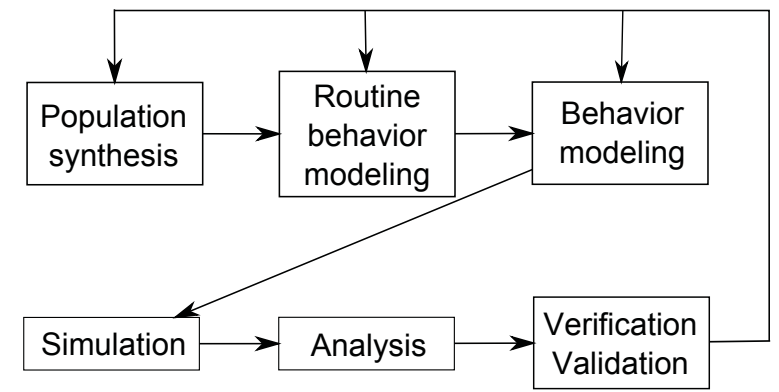

Figure 1: The workflow of the main components in POPSIM.

population synthesizer is used to generate the initial, static, properties of the agents in the population using statistics acquired from census bureaus or Subject Matter Experts (SME). Next, the routine behavior for each agent in the population is generated using activity statistics that may again originate from SMEs, which is typically the case when modeling populations of third-world countries. In recent years it has been shown that activity statistics can also be acquired, at least when modeling information societies, using crowdsourcing through mobile smart-phones [9]. In the subsequent step, domain or application specific behaviors, which represent all behaviors that are not considered routine, are added to the agents. Next, the population is simulated and the results are analyzed. Finally, during model development it is important that the model is properly verified and validated $(\mathrm{V} \& \mathrm{~V})$. This step is a challenging task in any simulation model requiring both qualitative and quantitative analysis of simulation results by developers, modelers, SMEs and end-users. The complexity of the task is even worse in agent-based models where simulations may result in emergent phenomena that are difficult to explain even for experienced SMEs [6]. Nevertheless, if the V\&V phase fails then the population modeler must iteratively revise the previous steps of the work-flow until satisfactory results are achieved.

Figure 2 illustrates a screen capture of the latest version of POPSIM. A map view is used to visualize city buildings, roads and cell phone coverage maps. Agents and their positioning are represented by color-coded blobs that are superimposed on the map. Grid-based intensity maps are used to highlight areas of the map that are densely populated (white) and areas that are not (gray). Bar- and line-plots are used to gain insight into the population's activities, healthstatus, opinion and so as time progresses in the simulation. In POPSIM, simulation ticks are converted into date and time as shown in the top-left corner of the screen-capture. POPSIM can be configured to execute slower than, faster than or in real-time depending on the requirements of the scenario or end-user as well as the computational complexity of the individual agents in the population.

\subsection{Population Synthesizer}

In POPSIM thousands of agents are simulated to represent the behavior of a real-world population. To incorporate behavioral diversity in the model each agent consists of a unique set of characteristic properties or parameters that primarily are used by the agent's reasoning mechanism to select the actions and activities that drives the agent towards its goals and self-interests. This modeling approach 


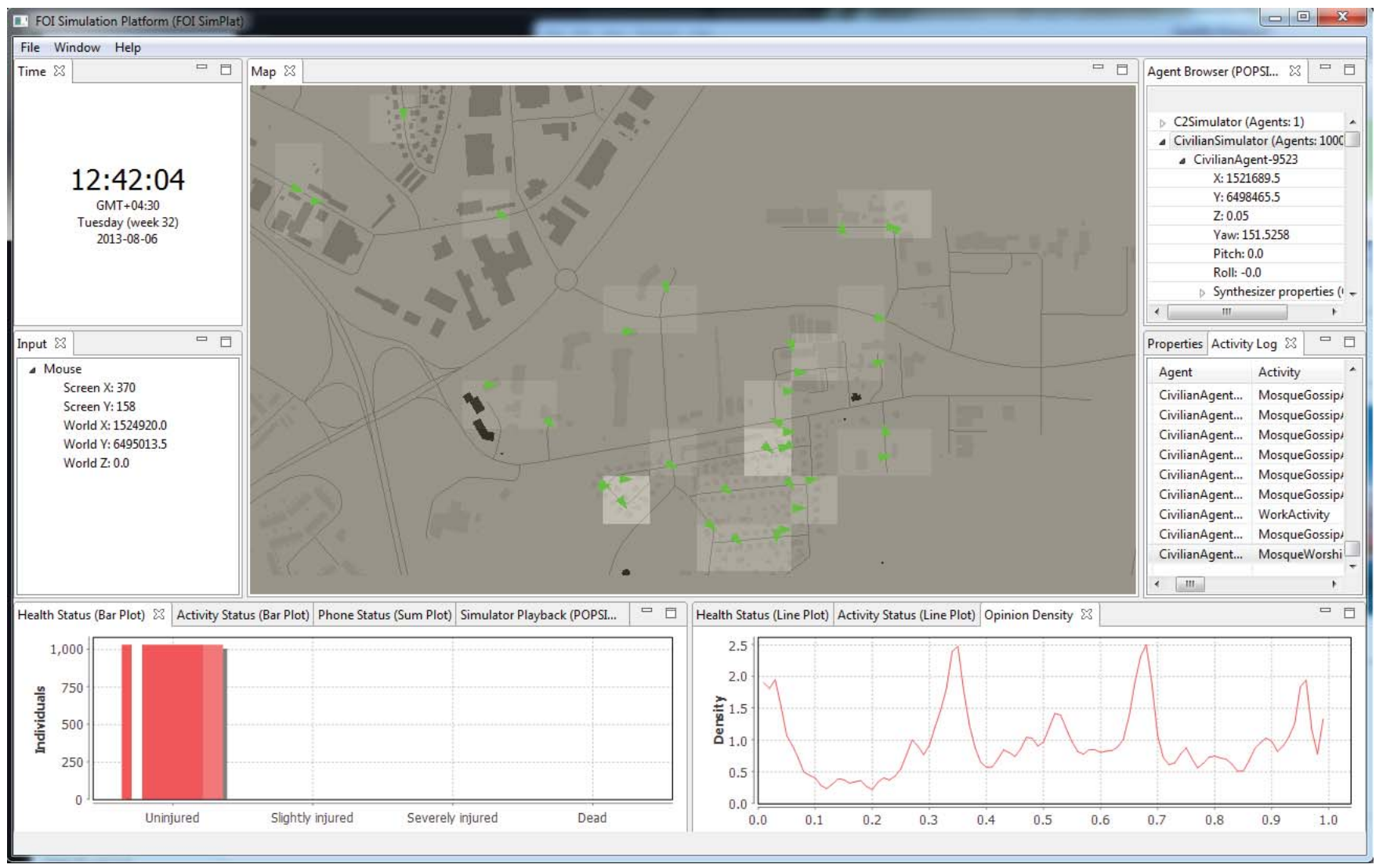

Figure 2: Screen capture of POPSIM.

requires, to ensure that POPSIM remains pragmatic from the modeler's perspective, that tools exists to assist in the initialization of these properties.

The population synthesizer embedded in POPSIM is based on previous works $[2,18,22]$. The tool is capable of fitting real-world statistics or data collected from either census bureaus or SMEs into probability distributions which in turn are randomly sampled to generate the initial state of the agent parameters. The tool supports a wide variety of distributions ranging from simple table-based distributions to relatively advanced multi-modal and multi-variate continuous distributions such as the Gaussian Mixture Model (GMM) [8].

The population synthesizer tool consists of a set of synthesizer modules each of which is responsible for creating agent properties using different types of input statistics or data. The following modules have been developed and added to the synthesizer tool:

- Demography synthesizer - Responsible for adding basic properties such as age, gender, occupation, etc.

- Geography synthesizer - Responsible for assigning geographical locations such as homes, workplaces, landmarks, etc. to the agents. The module pre-calculates all paths, based on shortest distance, between all locations known to the agent. Pre-calculating paths is an efficient method to reduce processing power, albeit increasing the memory requirements, allowing larger populations to be simulated.
- Relationship synthesizer - Responsible for generating relational bonds between agents. Common relationship types are family/households, friends or co-workers. Relations are used in POPSIM to, for instance, model information propagation or to model behaviors where parents drop-off and pick-up their kids at school or kindergarten on their way to and from work respectively.

- Opinion synthesizer - Responsible for generating the initial opinion and parameters values defined by the opinion update models from Section 3.3.5.

A screen capture of the synthesizer tool is provided in Figure 3. Bar-plots and kernel-density plots are used to show the distribution of a particular property of the synthesized population (in this case the occupation property is shown). Synthesizer templates are listed to the left representing the different modules and distributions mentioned above. The templates can be dragged-and-dropped into the main view that holds a tree-structure of template instances representing the population to be synthesized. The properties view on the bottom left is used to set all the parameter values as required by the selected template instance.

\subsection{Routine Behavior Modeling}

In the routine behavior modeling phase the agents are associated with plans representing activities of everyday behavior. In the current implementation of POPSIM daily plans are generated as follows. First, timestamp statistics 


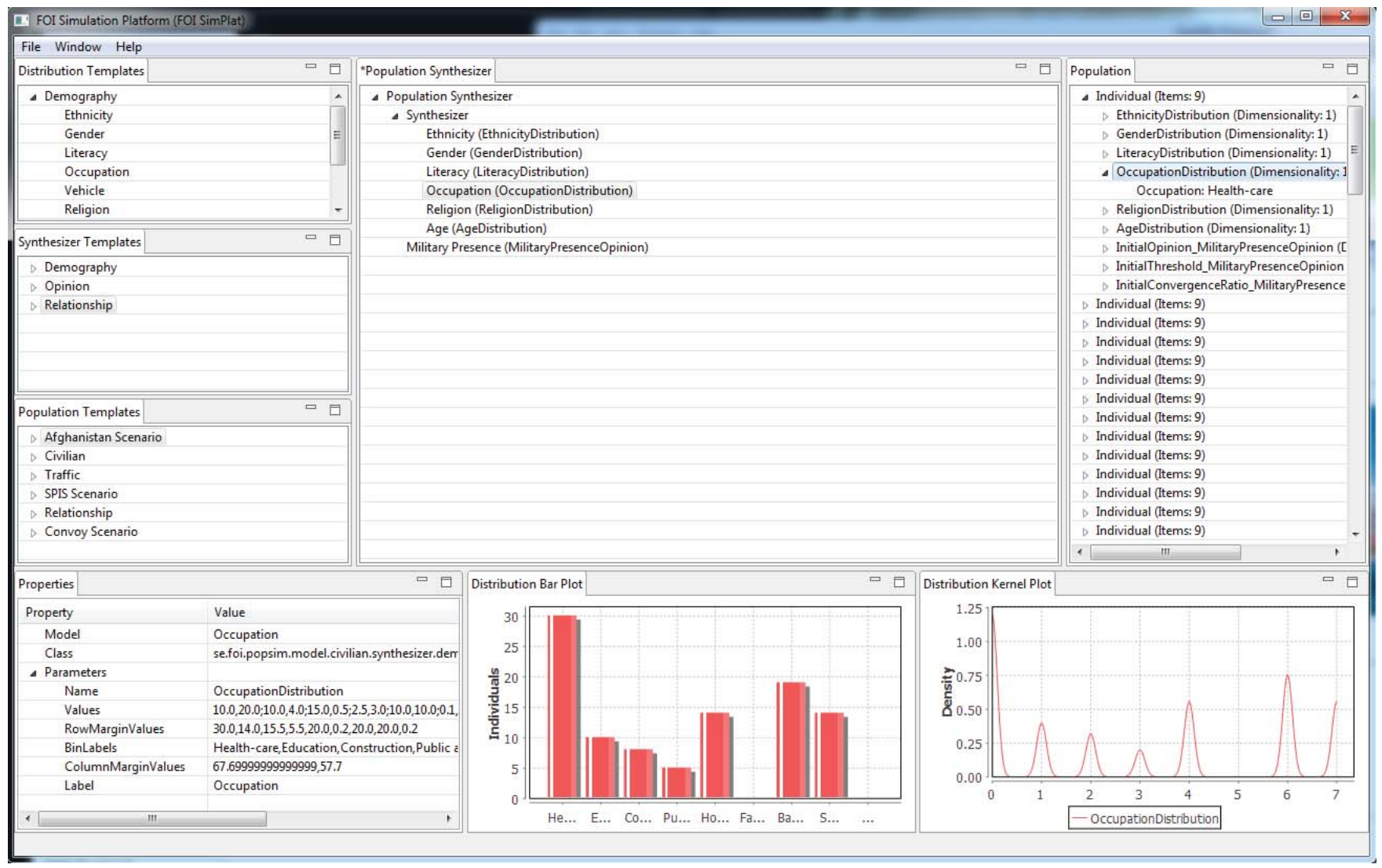

Figure 3: Screen capture of POPSIM's population synthesizer tool.

representing the real-world population's perception of morning, noon, afternoon, evening and night are collected using surveys or SMEs. A probability distribution is then fitted to the data and randomly sampled for each agent in the simulated population. In the next step, activity statistics are gathered mapping the discrete events (morning, noon, etc.) to activities such as working, eating, sleeping, etc. Again a probability distribution is fitted to the data and randomly sampled for each agent.

An activity within a plan is triggered to execute whenever the simulated time passes the timestamp associated with the activity. In Figure 4 an example of a population's routine behavior is plotted over time. The majority of the agents sleeps during night and starts work in the morning, eats at noon, resumes work in the afternoon and returns home for leisure time and eating in the evening.

\subsection{Infrastructure Models and Agent Services}

Let us now introduce the main infrastructure models and agent services developed in POPSIM starting with a brief description of activity and action modeling.

\subsubsection{Activity and Action Modeling}

In POPSIM an action represents the lowest level of behavior modeling. An action consists of Java code that defines the action's behavior and its end-condition. The action will execute continuously, once every simulation tick, until its end-condition is met. An activity is essentially a sequence of actions. When all actions in an activity have finished the

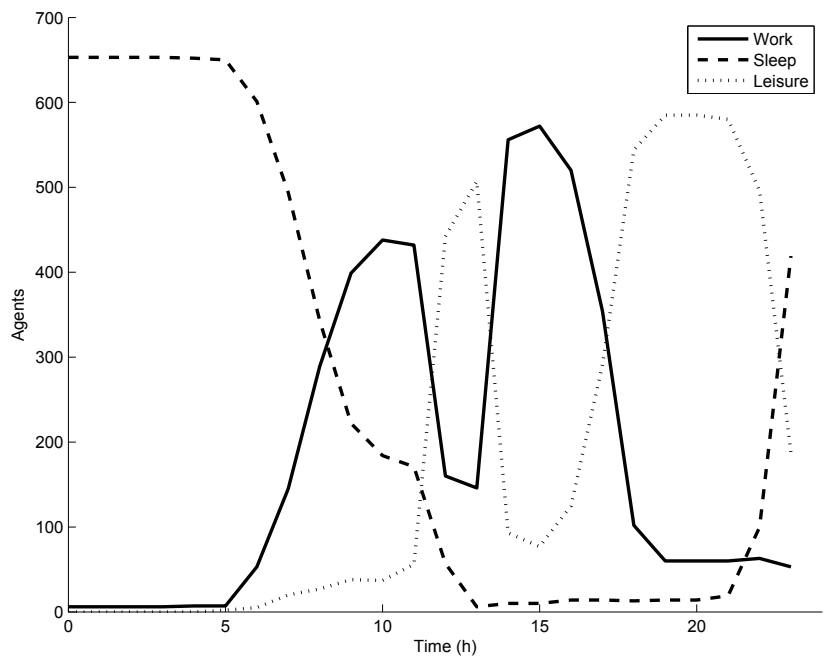

Figure 4: Example of a routine behavior plotted over time.

activity is finished.

POPSIM includes an activity processing service which is used to manage the execution of activities and actions. The service is used by the agent's reasoning mechanism which is responsible for selecting activities to execute.

\subsubsection{Field-of-View (FOV)}

The FOV function, which is used to locate neighboring 
agents in the simulation, is a critical function in any ABM implementation. In POPSIM the FOV function was implemented using the approach found in [20] which is illustrated in Figure 5. Neighboring agents are those that are located within the grayed-out area in the figure.

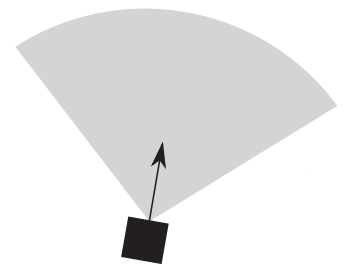

Figure 5: Field of view (FOV) of an agent. Agents within the grayed-out area are visible to the agent.

\subsubsection{Communication}

Agents in POPSIM can communicate with each other directly whenever the agents are within each other's FOV or remotely using simulated cell phones assuming that the caller and the receiver are both connected to a base-station. Equation 1 is used to determine if a cell-phone has reception or not.

$$
\begin{gathered}
\text { reception }= \begin{cases}\text { true, } & \text { if } \frac{S}{N}>\text { threshold } \\
\text { false, } & \text { otherwise }\end{cases} \\
S=\frac{P_{t} \times G_{t} \times G_{r}}{\frac{(4 \pi \times r)^{2}}{\lambda^{2}}} \\
N=\sum_{k=1}^{K} n_{k}
\end{gathered}
$$

, where $S$ and $N$ represent signal and noise respectively. $S$ is calculated using $P_{t}$ which is the effect used by the basestation, $G_{t}$ and $G_{r}$ which represent the antenna gain at the base-station and cell-phone respectively, $r$ which is the distance between base-station and cell-phone and $\lambda$ which is the wavelength of the transmitted signal. $N$ is calculated by summing the emitted noise, $n_{k}$, of all noise sources, $K$. Noise sources can be added to model either as background noise ( $k T B F$-noise) or as noise introduced by one or more jamming devices. Noise originating from a jamming device is calculated using a variant of the formula used to calculate $S$.

POPSIM employs the JADE framework to represent messages following the Foundation for Intelligent Physical Agents (FIPA) specification [3].

\subsubsection{Movement, Transportation and Route-Planning}

Road-networks are the primary means in which agents can move from one point to another within POPSIM. Roadnetworks are modeled in POPSIM using line-based GIS databases where we have assumed that each line in the database represents a two-way road segment. The GIS database is automatically converted into graphs that in turn can be used by route-planning algorithms to, for instance, search for the shortest path between two arbitrary points in the network.
Given the path calculated by the route-planner an agent can be instructed to move along the path using the Move activity. To achieve realistic movement patterns, at least on an aggregated level, we have similarly to [19] implemented the following speed governing rules that are executed by the agents while moving:

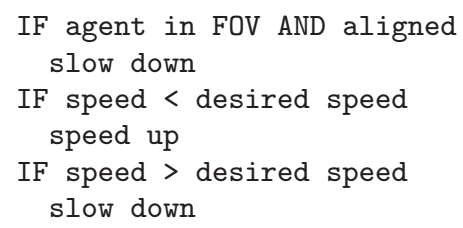

Using these simple rules an agent is able to maintain its desired speed while at the same time prioritizing the distance to other agents on the road-network. Emergent traffic congestion occurs naturally when two densely populated road-segments joins into one. Furthermore, our agents are programmed to follow the three second rule, hence, the distance between agents on high-speed road segments will be longer compared to low-speed segments.

\subsubsection{Opinion Modeling}

In POPSIM an opinion represents what an agent thinks or feels in a particular topic or question of interest which ultimately affects the behavior of the agent. Typical examples of opinions are political views, regime popularity, attitudes towards the presence of police or military troops, and so on.

We model opinions and the dynamics of opinions using well-known methods derived from the social simulation and complex systems research societies. In the current version of POPSIM we have implemented the following continuous models: 1) the Deffaunt model [7]; and 2) the HegselmannKrause model [13]. In POPSIM the Deffaunt model is used to update opinions whenever individual agents meet in smaller groups (e.g. friend gatherings, family celebrations) whereas the Hegselmann-Krause model is used where agents meet at formal meetings (e.g. religious/cultural events or political meetings). Refer to [16] for an excellent introduction and review of the opinion models used in POPSIM.

\section{PRACTICAL APPLICATIONS}

To demonstrate one out of several potential real-world applications of POPSIM we have in previous works developed a scenario targeting end-users within the crisis and disaster management community [15]. In the scenario a toxic chemical substance is released by accident in the downtown area of a city resulting in a major disaster affecting thousands of individuals. The scenario was implemented using POPSIM and empirical results were provided to gain insight into the number of affected individuals and their severity of injury while considering the intangible factors of human behavior in addition to physical factors such as the size and shape of buildings and wind direction.

POPSIM has also been used in the past to demonstrate the concept of Social Coverage Maps (SCM) which is a visual representation of how localized disruptions in, for instance, city infrastructure may affect the society as a whole [14]. The application was inspired by the San Francisco incident in 2011 where transit authorities decided to cut off wireless service at a number of train stations to prevent protests. Although the intent was to secure public safety their actions 
resulted in even more protests ultimately worsening the situation.

\section{CONCLUSIONS AND FUTURE WORK}

Even though population models are fairly common in simulation applications there is a lack of general tools or platforms that can be used to support the modeling work. In this paper we have introduced POPSIM which is an extensible platform that encapsulates several fundamental components required to efficiently create human population models. The platform employs AI techniques such as ABM, rule-based systems, path-planning algorithms, etc. to model agent behaviors. The platform also provides: basic infrastructure models to support execution of agent activities; a populations synthesizer tool to generate the initial state of the population; and a wide range of visualization possibilities that can be used to analyze simulation runs in real-time or offline.

Future works include, besides extending and improving the core functionality of the platform, exploring additional application domains. The POPSIM platform is, for instance, currently being used to support military training targeting military operations in urban terrain (MOUT). The main goal of the training tool is to enhance the trainee's ability to trade-off situational awareness vs. risk while considering, in addition to threats from irregulars, intangible human factors such as the civilian population's attitude or opinion towards the military forces.

\section{Acknowledgment}

This work was supported by the FOI research project "Synthetic Actors", which is funded by the R\&D programme of the Swedish Armed Forces.

\section{REFERENCES}

[1] M. Balmer, M. Rieser, K. Meister, D. Charypar, N. Lefebvre, K. Nagel, and K. Axhausen. MATSim-T: Architecture and simulation times. Multi-Agent Systems for Traffic and Transportation Engineering, pages 57-78, 2009

[2] R. J. Beckman, K. A. Baggerly, and M. D. McKay. Creating synthetic baseline populations. Transportation Research Part A Policy and Practice, 30(6):415-429, 1996.

[3] F. Bellifemine, A. Poggi, and G. Rimassa. JADE - a FIPA-compliant agent framework. In Proceedings of the Practical Applications of Intelligent Agents, 1999.

[4] A. Bier, M. Bernard, G. Backus, M. Glickman, and S. Verzi. Using a hybrid cognitive-system dynamics model to anticipate the influence of events and actions on human behaviors. Proceedings of the 29th International Conference of the System Dynamics Society, July 2011.

[5] L. Bloomberg and J. Dale. Comparison of VISSIM and CORSIM traffic simulation models on a congested network. Transportation Research Record 1727, TRB, National Research Council, pages 52-60, 2000.

[6] E. Bonabeau. Agent-based modeling methods and techniques for simulating human systems. Proceedings of the National Academy of Sciences of the United States of America, 99(10):7280-7287, 2002.
[7] G. Deffuant, D. Neau, F. Amblard, and G. Weisbuch. Mixing beliefs among interacting agents. Advances in Complex Systems, 3:87-98, 2000.

[8] R. O. Duda, P. E. Hart, and D. G. Stork. Pattern Classification (2nd Edition). Wiley-Interscience, 2000.

[9] N. Eagle and A. Pentland. Reality mining: sensing complex social systems. Personal Ubiquitous Comput., 10(4):255-268, Mar. 2006.

[10] J. Forrester. Industrial dynamics: A major breakthrough for decision makers. Harvards business review, 36(4):37-66, 1958.

[11] A. Grignard, P. Taillandier, B. Gaudou, D. Vo, N. Huynh, and A. Drogoul. GAMA 1.6: Advancing the art of complex agent-based modeling and simulation. In PRIMA 2013: Principles and Practice of Multi-Agent Systems, volume 8291 of Lecture Notes in Computer Science, pages 117-131. Springer Berlin Heidelberg, 2013.

[12] S. Gwynne, E. Galea, P. Lawrence, and L. Filippidis. Modelling occupant interaction with fire conditions using the buildingEXODUS evacuation model. Fire Safety Journal, 36(4):327-357, 2001.

[13] R. Hegselmann and U. Krause. Opinion dynamics driven by various ways of averaging. Computational Economics, 25(4):381-405, 2005.

[14] M. Jändel, S. Lindquist, and L. J. Luotsinen. Social coverage maps. The 10th International Conference on Informations Systems for Crisis Response and Management (ISCRAM), pages 241-230, may 2013.

[15] P. Leffler, C. Carling, E. Carlson, L. J. Luotsinen, O. Björnham, J. Burman, R. Forsgren, B. Koch, F. Näsström, O. Parmhed, and C. Troeng. A simulation based crisis management laboratory (SIMBRA). The 11th International Symposium on Protection against Chemical and Biological Warfare Agents, June 2013.

[16] J. Lorenz. Continuous Opinion Dynamics under bounded confidence: A Survey. International Journal of Modern Physics C, 18(12):1819-1838, 2007.

[17] S. Luke, C. Cioffi-Revilla, L. Panait, K. Sullivan, and G. Balan. MASON: A Multiagent Simulation Environment. Simulation, 81(7):517-527, July 2005.

[18] R. Moeckel, K. Spiekermann, and M. Wegener. Creating a synthetic population. 8th International Conference on Computers in Urban Planning and Urban Management (CUPUM), May 2003.

[19] K. Nagel and M. Rickert. Parallel implementation of the TRANSIMS micro-simulation. Parallel Computing, 27(12):1611-1639, 2001.

[20] C. W. Reynolds. Steering behaviors for autonomous characters. Game Developers Conference $(G D C)$, pages 763-782, 1999.

[21] J. A. Sokolowski and C. M. Banks. Modeling complex social behavior: A system dynamics approach. Proceedings of the 19th Conference on Behavior Representation in Modeling and Simulation, 2010.

[22] X. Ye, K. Konduri, R. M. Pendyala, B. Sana, and P. Waddell. A methodology to match distributions of both household and person attributes in the generation of synthetic populations. 88th Annual Meeting of the Transportation Research Board, Jan. 2009. 